\title{
Competencias cognitivas, procedimentales y actitudinales en ciencias naturales de los estudiantes de grado 4 to y 5 to de primaria
}

\author{
The cognitive, procedural and attitudinal skills in natural sciences of students in the \\ 4 to and 5to grades
}

\author{
Gisele Carolina Pabón Guevara \\ caritopg625@gmail.com \\ Código ORCID: 0000-0001-8387-6881 \\ Universidad Metropolitana Educación, Ciencia y Tecnología \\ (Umecit), Ciudad de Panamá, Panamá
}

| Artículo recibido en marzo 2021

| Arbitrado en abril 2021

| Aceptado en abril 2021

Publicado en mayo 2021

\section{Resumen}

La presente investigación tiene como objetivo, describir las competencias cognitivas, procedimentales y actitudinales en ciencias naturales de los estudiantes de los grados cuarto y quinto de la institución educativa Gabriel García Márquez del municipio de Soacha, departamento de Cundinamarca. Se considera una investigación de tipo descriptiva, con un diseño de campo, transeccional contemporáneo univariable. La población estuvo conformada por 102 estudiantes: 52 de cuarto y 50 de quinto de la institución estudiada. Para la recolección de los datos se empleó como instrumento una evaluación de las competencias cognitivas, procedimentales y actitudinales basada en las pruebas "Saber", contentiva de 40 ítems de alternativas de respuestas múltiples. La validez se realizó mediante la técnica de validación, por tres expertos en ciencias y la confiabilidad se calculó mediante el Alfa de Cronbach, obteniéndose un valor de 0,75 lo que indica que el instrumento es altamente confiable. Para el análisis de los resultados se utilizó la estadística descriptiva; específicamente la frecuencia absoluta y porcentual, y la mediana como medida de tendencia central por cuanto la variable se midió en un nivel ordinal. Los resultados indican que los estudiantes poseen predominio de las competencias procedimentales en relación con las competencias científicas.

\begin{abstract}
This research aims to describe the cognitive, procedural and attitude skills in natural sciences of students in the fourth and fifth grades of the Gabriel García Márquez educational institution in the municipality of Soacha, Cundinamarca department. It is considered a descriptive type research, with a field design, unique contemporary transactional. The population consisted of 102 students: 52 fourth and 50 fifth from the institution studied. For the collection of data, an assessment of cognitive, procedural and attitudinal competencies based on the "Saber" tests was used as an instrument, contentive of 40 items of multi-response alternatives. The validity was done using the validation technique, by three science experts and reliability was calculated using the Cronbach Alpha, obtaining a value of 0.75 indicating that the instrument is highly reliable. Descriptive statistics were used for the analysis of the results; specifically, absolute and percentage frequency, and median as a central trend measure as the variable was measured at an ordinal level. The results indicate that students have a predominance of procedural competencies in relation to scientific competencies.
\end{abstract}

Palabras clave:

Competencias científicas; competencias cognitivas; competencias procedimentales; competencias actitudinales

Keywords:

Scientific competences, cognitive competences, procedural competencies, attitudinal competencies 


\section{INTRODUCCIÓN}

La educación se reconoce como un proceso inherente al ser humano. La curiosidad y la búsqueda de respuestas son propias del ser humano. Las personas desde el nacimiento se educan, es así como la familia se convierte en la primera institución, que proporciona las herramientas básicas para comprender el mundo y desenvolverse en él.

Con el pasar de los años la sociedad invita a ingresar a la educación formal, la cual tiene como objetivo formar al ciudadano para que responda a las necesidades e intereses del tiempo y el espacio en el cual se desarrolla. Es así que la educación está íntimamente relacionada con la humanidad. Esta realidad es fácil precisarla, debido a los numerosos cambios y adelantos que ha tenido la sociedad moderna, gracias a las innovaciones tecnológicas logradas por el avance de las ciencias.

Según Luengo (2004), etimológicamente la palabra educación proviene de los verbos latinos educere, que significa "conducir fuera"; es decir, sacar las habilidades y potencialidades que se encuentran en el interior del ser humano, y educare que equivale a "criar", donde se le da importancia al medio en el cual se desenvuelve el individuo, ya que es allí donde se adquieren las herramientas y conocimientos requeridos.

A pesar de que estas posiciones propuestas por Luengo (2004) de moldear a la persona y proporcionar conocimientos, estuvieron separadas y cómo se evidencia a través del tiempo con las teorías que se han formado alrededor de la educación, hoy se sabe que es tan importante el contexto, como las potencialidades que posee el individuo en su interior, y que el fin de la educación debe ser proporcionar un medio y los recursos adecuados para potenciar las habilidades que tiene cada persona.

En la búsqueda constante por cumplir con el objetivo de la educación, se han propuesto enfoques y teorías dirigidos a mejorar los procesos de enseñanza aprendizaje, como son el método tradicional o conductismo, orientado a moldear el comportamiento del individuo, hasta llegar a enfoques más humanistas como el aprendizaje significativo y el constructivismo; entre otros, los cuales han realizado aportes importantes para la educación actual, como la educación por competencias, que aunque parece un término relativamente reciente, ha estado presente desde la antigua Grecia y se ha nutrido con diferentes enfoques y corrientes.

De esta forma, los organismos internacionales encargados de la educación tal como la Unesco, (2009) plantea que las competencias científicas son una cultura científica que forma ciudadanos, para que puedan responder a los avances científicos y tecnológicos del mundo actual, además de actitudes responsables que les permitan resolver problemas cotidianos del entorno y donde la escuela primaria siente las bases para cumplir con este fin.

En Colombia la preocupación por una educación de calidad ha llevado a que el Ministerio de Educación, defina estándares básicos y lineamientos básicos (2004), los cuales, entre otras cosas, establecen que los estudiantes de los grados cuarto y quinto, al terminar ese ciclo de formación, deben no sólo conocer contenidos de ciencia; es decir tener competencias cognitivas, sino también saber hacer (Competencias procedimentales), además de tener actitudes positivas hacia las ciencias.

En tal sentido, Tobón (2013) define las competencias desde un enfoque socio formatico, lo que da una visión integral del ser humano, ya que las competencias no sólo encierran los conocimientos y procedimientos para realizar algunas tareas, sino que tiene en cuenta de manera relevante a la persona, la esencia humana, los valores éticos y la forma cómo se resuelven los problemas a partir del contexto, transformándose y transformándolo.

De esta manera, en Colombia según mandato del Ministerio de Educación Nacional 
se realizan las pruebas Saber, las cuales se orientan a realizar una evaluación de las competencias que los estudiantes han adquiridos en su formación de ciencias naturales en los grados 3, 5, y 9. Estas pruebas son organizadas y monitoreadas por el Instituto Colombiano para el fomento de la educación superior (Icfes). Los resultados de estas pruebas en los años 2014 y 2016 con referencia a las competencias en ciencias naturales indican que existe un nivel de desempeño mínimo, a tal punto que solo el $65 \%$ de los estudiantes se ubicaron en un nivel de desempeño medio en el área.

Esta situación es similar en la institución educativa Gabriel García Márquez, en donde se observa que los estudiantes de grado cuarto tienen grandes dificultades en el diseño de experimentos y en la comprensión de los fenómenos naturales que ocurren en su alrededor.

Es por tanto que se plantea en este estudio conocer ¿cuáles son las competencias cognitivas, procedimentales y actitudinales en Ciencias Naturales de los estudiantes del grado 4 de la institución educativa Gabriel García Márquez de Soacha?

\section{Fundamentación teórica}

El concepto de competencias es muy antiguo, según Hernández (2005) los orígenes de la idea de competencia inician en Grecia con Aristóteles, quien pensaba que el ser tiene dos modalidades el acto y la potencia. El ser en acto son las propiedades con las que cuenta cada ser humano y dentro de él está un ser en potencia, que nombra lo que algo a alguien es capaz de ser o hacer.

Del mismo modo, Tobón (2013) indica que el término competencia se ha nutrido a través de la historia con los aportes de diversas ciencia y especialistas, entre ellos, por nombrar algunos: la filosofía griega, la competencia lingüística propuesta por Chomsky, la competencia comunicativa de Hymes y Gumperz, la psicología cognitiva, la educación para el trabajo donde se definen las competencias para el área laboral como forma de aumentar la productividad y para la pedagogía en el campo de la educación.

El término competencia en Colombia se relacionó con la educación, según lo expuesto por Tobón (2013) en la década de los 60, se puso en práctica en los niveles educativos en los años 90 y entró como política educativa a partir del año 2000. También Parra (2018, p.4) indican que en educación:

El término competencia ha tenido diferentes cambios importantes, que le han dado diversos significados: el primero se refirió a conductas, propias del enfoque conductista; el segundo a como se muestra el control del conocimiento sobre las acciones; el tercero son acciones que se muestran eficaces al materializarse; el cuarto significado es la integración de conductas, conocimientos, habilidades, destrezas, nivel de eficacia y eficiencia $y$ el quinto significado es una visión holística que tiene en cuenta el saber, el saber hacer, saber actuar, saber convivir y saber hacer para enfrentarse al mundo actual.

Otras perspectivas más contemporáneas son las de Hernández (2005), para quien en forma general la competencia se define como el conjunto de saberes, capacidades y disposiciones que hacen posible actuar e interactuar de manera significativa en contextos determinados. Para Tobón (2013), las competencias son las formas en que la persona interactúa y trasforma el contexto, ya que éste genera situaciones que impulsan a actuar.

En el espacio colombiano y desde el ámbito académico, el Ministerio de Educación Nacional (1994 c.p. Vallejo, 2014) define competencia como el conjunto integrado de conocimientos (Saber-Cognitivo), habilidades (Hacer-Procedimental) y actitudes (Ser-Actuar) 
que debería tener una persona que pase por el sistema educativo colombiano.

Asimismo, se pueden encontrar conceptos de diferentes enfoques; como, por ejemplo, el socio formativo que define competencias según Tobón (2013) como actuaciones integrales para identificar, interpretar, argumentar y resolver problemas del contexto, desarrollando y aplicando de manera articulada diferentes saberes (saber ser, saber convivir, saber hacer y saber conocer) con idoneidad, mejoramiento continuo y ética.

El mismo autor también considera que el enfoque socio-formativo de competencias incluye: inteligencias múltiples, actitudes, destrezas, objetivos, propósitos, capacidades y habilidades, dando una visión integral del concepto de competencia que tiene en cuenta todas las dimensiones del ser humano; por lo cual, como afirma el autor citado las competencias no son sólo observables, sino una compleja estructura de atributos necesarios para el desempeño en diversas situaciones.

Es así como los diferentes aportes al concepto de competencias, lo han nutrido de tal manera que dan una visión holística de las mismas, donde se tienen en cuenta todas las dimensiones del ser humano, puesto que como expone Tobón (2013) sería una concepción reduccionista de las competencias, centrarse sólo en el saber hacer, dejando de lado el entender, el comprender, los valores y la interacción.

En cuanto a las competencias científicas, al igual que las competencias en general, éstas tienen diferentes definiciones según la visión y los intereses de las entidades que las promulgan. Para Hernández (2005, p. 21) "Las competencias científicas serían el conjunto de saberes, capacidades y disposiciones que hacen posible actuar e interactuar de manera significativa en situaciones en las cuales se requiere producir, apropiar o aplicar comprensiva $y$ responsablemente los conocimientos científicos."
De igual manera el autor antes citado indica que las competencias en ciencias con un enfoque social comprenden diversas capacidades, entre ellas las de: Asumir la dimensión social de las ciencias como prácticas de comunidades; examinar críticamente los usos sociales de las ciencias; establecer conexiones entre técnica, ciencia y sociedad; intercambiar flexiblemente conocimientos y puntos de vista; llevar los valores científicos a la vida social teniendo en cuenta el contexto.

La Organización para la Cooperación y Desarrollo Económico (OCDE) (2006, c.p. Vallejo 2014, p.32) menciona que el Programa Internacional de Evaluación a Estudiantes (PISA) entiende la competencia científica como aquella que "incluye los conocimientos científicos y el uso que de estos conocimientos haga un individuo para identificar preguntas, adquirir nuevos conocimientos, explicar los fenómenos científicos y sacar conclusiones basadas en evidencias, sobre asuntos relacionados con la ciencia"

Según expone Vallejo (2014), en Colombia, país que asumió las competencias científicas como un enunciado en el Plan Sectorial de Educación (2010 - 2014), como competencias básicas que los estudiantes deben desarrollar en su proceso escolar, se habla públicamente de ese concepto desde 1994 cuando se organiza una comisión de sabios colombianos, los cuales tenían la misión de establecer la relación entre Ciencia-Educación-Desarrollo.

Mora y Parga (2005) expresan que el ingreso de las competencias en la evaluación de los aprendizajes escolares viene patrocinada desde políticas internacionales del Banco Mundial, orientadas a alcanzar grados de competitividad deseables y sostenibilidad ambiental, lo cual es ratificado por Vallejo (2014), quien menciona que el enfoque de competencias científicas en la educación colombiana ha sido el resultado de la adopción de políticas internacionales y 
la adaptación de estas al contexto educativo nacional.

Para el Ministerio de Educación Nacional, como lo expresa Vallejo (2014), la educación científica escolar debe desarrollar tres competencias: producir y manejar conocimiento científico; generar bienes sociales y económicos para la sociedad, además de estudiar, explicar, razonar y argumentar los impactos negativos y/o positivos de la ciencia y la tecnología. Lo que pone en evidencia el alto alcance que tiene para el órgano rector del país este concepto $y$, con ello, la importancia de esta investigación.

Bajo esa premisa y para lograr la implementación de las competencias en Colombia, el Ministerio de Educación Nacional (MEN), tal y como lo indica Vallejo (2014), en 1998 publica los lineamientos curriculares, los cuales establecen el horizonte epistemológico y pedagógico de la educación basada en competencias, definiendo los parámetros para su implementación.

Los contenidos conceptuales o el saber conocer, según Tobón (2013, p. 250) se definen como "Un conjunto de herramientas necesarias para procesar la información de manera significativa según las expectativas individuales, las propias capacidades y los requerimientos de una situación en particular". Esta conceptualización al referirse al procesamiento significativo, lo que involucra el desarrollo de procesos cognitivos, se aleja suficientemente de la educación tradicional, donde prevalece la repetición mecánica de contenidos.

Tradicionalmente se tiene la concepción de que memorizar contenidos es tener conocimiento, pero el crecimiento de conceptos y teorías a través de la historia hacen este proceso cada vez más difícil, por lo que hoy en día cuando se habla de competencias conceptuales, según Tobón (2013 p. 250), se hace énfasis "En la formación de habilidades y estrategias para que las personas puedan aprender a procesar y a manejar dicho conocimiento sin necesidad de memorizarlo, mediante procesos de indagación sistemática, análisis crítico, clasificación, elaboración, reconstrucción y aplicación de la información"

Al respecto, Pozo y Gómez (2009) también explican que lograr la comprensión de contenidos abstractos y generales, se realiza mediante contenidos específicos; pudiendo ser además, los contenidos conceptuales o verbales de varios tipos, entre ellos los siguientes: datos, conceptos y principios.

Para Pozo y Gómez (2009, p. 86) "Los principios serían conceptos muy generales, de un gran nivel de abstracción, que suelen subyacer a la organización conceptual de un área, aunque no siempre se hagan lo suficientemente explícitos". Mientras que un dato o hecho, según explican Pozo y Gómez (2009, p.85) "Es una información que afirma o declara algo sobre el mundo". Para comprender un dato se requiere de una red de conceptos que explique por qué se produce y qué consecuencias tiene.

Respecto a la forma de evaluar las competencias conceptuales, Castillo y Cabrerizo (2010), mencionan que es el ámbito más conocido por los docentes, debido a que la actividad evaluadora en la escuela, tradicionalmente se basa específicamente en estas competencias, y se dejan de lado las competencias procedimentales y actitudinales, por lo cual la mayoría de las veces la evaluación no resulta ser integral.

Con respecto al campo de la ciencia, Pozo y Gómez (2009) mencionan que para esa área, según las actuales concepciones sobre naturaleza y epistemología, son un proceso histórico y social, en el cual no se pueden dejar de lado los contenidos procedimentales, ya que con ayuda de ellos es como se han construido los conocimientos y teorías científicas que se tienen hoy en día.

Con relación al saber hacer o competencias procedimentales, según Tobón (2013, p. 251) 
"Consiste en desempeñarse en la realización de una actividad o en la resolución de un problema comprendiendo el contexto y teniendo como base la planeación". Por su parte, Pozo y Gómez (2009) opinan que el conocimiento procedimental, se adquiere eficazmente a través de la acción y se ejecuta de modo automático, sin tener consciencia de ello.

De esta manera, el desarrollo del conocimiento procedimental es muy relevante en todas las áreas, pero más aún en las ciencias por sus características prácticas y para esta investigación por ser su objeto de estudio. De allí que sean muy importantes las consideraciones de Castillo y Cabrerizo (2010), quienes expresan que proporciona a los estudiantes herramientas y habilidades que les permiten construir su propia experiencia social y desplegar el trabajo intelectual.

Del mismo modo, Pozo y Gómez (2009) explican que existen cuatro fases principales para la adquisición de procedimientos: 1 . Declarativa o de instrucciones, 2. Automatización o consolidación, 3. Generalización o transferencia del conocimiento y 4 . Transferencia del control.

La fase declarativa o de instrucciones como su propio nombre indica y como mencionan Pozo y Gómez (2009), tiene como objetivo explicar o describir la secuencia de acciones o pasos, que debe realizar un estudiante para diseñar diferentes estrategias que le permitan contrarrestar diversas explicaciones que recibe de parte del instructor o docente.

En la fase de automatización, Pozo y Gómez (2009) expresan, que el estudiante debe poner en práctica repetidamente las secuencias de la fase declarativa con supervisión del docente, para que al final cuando el aprendiz ya domina las técnicas de una tarea, pueda realizar las fases de transferencia de conocimiento y transferencia de control.

Según Pozo y Gómez (2009) para introducir al estudiante en las técnicas complejas de la ciencia, se le debe instruir en procedimientos que son producto de una larga construcción cultural. Los procedimientos generales que debe aprender un estudiante son leer y comprender textos, decodificar gráficas, comunicar sus ideas y conocimientos.

Para Pozo y Gómez (2009) es necesario dar relevancia a los contenidos procedimentales en el currículo, lo que implicaría dedicar más tiempo para ellos en las clases de ciencias lo cual no se hace, sin embargo, hay que considerar que los contenidos procedimentales desempeñan un papel secundario, acompañando y facilitando los contenidos conceptuales sin tener una estructura y un objetivo propio.

De igual manera, Pozo y Gómez (2009) alertan sobre la relevancia que se dé a los contenidos procedimentales, ya que existe el riesgo, muy cierto y factible, de tomar la enseñanza de procedimientos en la ciencia, como un modo para aplicar y demostrar conocimientos más que para generarlos y construirlos, lo que sin lugar a dudas no es lo ideal.

Respecto a la forma de evaluar Castillo y Cabrerizo (2010), afirman que ésta debe realizarse desde la práctica de los mismos procedimientos en diferentes situaciones $y$, que debe estar integrada con los conocimientos conceptuales y actitudinales. Para esto se requiere el uso de pruebas o ejercicios, en donde los estudiantes puedan poner en práctica los procedimientos adquiridos en situaciones conocidas y nuevas.

En cuanto a las competencias actitudinales o el saber ser, Tobón (2013) menciona que consisten en la articulación de diversos contenidos afectivo-motivacionales, que se caracterizan por la construcción de la identidad personal, así como la conciencia y control del proceso emocional-actitudinal para la realización de una actividad o resolución de un problema.

Para Delors (1996 c.p. Tobón 2013) desde el saber ser o competencia actitudinal, 
se promueve la convivencia ciudadana para que las personas asuman sus derechos $y$ deberes, con responsabilidad y buscando la construcción de una sociedad civil, democrática y solidaria. Concepto muy importante para esta investigación porque define la formación de un estudiante en su esencia valorativa de manera integral.

Bajo perspectivas muy semejantes, Pozo y Gómez (2009) consideran que las actitudes se refieren a reglas o patrones de conducta; es decir, disposiciones que llevan a comportarse de modo consciente en una situación determinada. De este modo, el objetivo de la educación en actitudes es lograr cambios en las capacidades autónomas y valores, hacer que los estudiantes interioricen como valores ciertas normas $y$ formas de comportarse; por ejemplo, responsabilidad en la entrega de un trabajo, respeto ante opiniones diferentes o tolerancia hacia los otros.

Como se observa de las posiciones anteriormente explicadas, las actitudes en el campo educativo son tan importantes como las procedimentales o las cognitivas. Lo que ratifican Castillo y Cabrerizo (2010, p. 146) al opinar que "La importancia que adquieren las actitudes en el ámbito educativo está justificada desde el momento en que desde todas las áreas del currículum pueden enseñarse y trasmitirse actitudes $y$ valores junto a conceptos $y$ estrategias."

En este sentido, Tobón (2013) indica que el saber ser se compone de valores, actitudes y normas, siendo los valores procesos cognitivosafectivos perdurables, que se orientan hacia metas ideales; en cuanto a las actitudes, estas con componentes actitudinal, cognitivo y afectivo, se aprenden en la interacción social y por último están las normas, las cuales dan las pautas esenciales para vivir en sociedad y relacionarse con las personas y el contexto.

Al respecto, Pozo y Gómez (2009) exponen que el modelado o aprendizaje por imitación es uno de los más importantes mecanismos para que los estudiantes adopten actitudes. Este procedimiento requiere elementos afectivos, representacionales e identificación con el modelo. La pertenencia a un grupo social de referencia y la identificación al igual que los procesos de conformidad a la presión grupal, hacen que la persona tienda a adaptarse a las normas y actitudes expuestas por el grupo mayoritario.

Las palabras expresadas por Pozo y Gómez en el párrafo anterior dan cuenta del papel protagónico de los grupos para la formación de valores, sobre todo en los niños y adolescentes; sin embargo, aunque la escuela asume la enseñanza de actitudes y por lo tanto valores, se debe tener en cuenta que los valores y actitudes que los estudiantes poseen y muestran en el aula, según Castillo y Cabrerizo (2010) son los que provienen del exterior de las aulas y se construyen mediante procesos socioculturales del contexto, incluyendo los adquiridos en el hogar.

\section{MÉTODO}

La presente investigación se considera de tipo descriptiva, con un diseño de campo, transeccional contemporáneo univariable. La población estuvo conformada por 102 estudiantes: 52 de cuarto y 50 de quinto de la institución estudiada. Para la recolección de los datos se empleó como instrumento una evaluación de las competencias cognitivas, procedimentales y actitudinales basada en las pruebas "Saber", contentiva de 40 ítems de alternativas de respuestas múltiples. La validez se realizó mediante la técnica de validación, por tres expertos en ciencias y la confiabilidad se calculó mediante el Alfa de Cronbach, obteniéndose un valor de 0,75 lo que indica que el instrumento es altamente confiable. Para el análisis de los resultados se utilizó la estadística descriptiva; específicamente la 
frecuencia absoluta y porcentual, y la mediana como medida de tendencia central por cuanto la variable se midió en un nivel ordinal. Los datos se presentan en tablas y gráficos con su respectiva interpretación. Para ello se calculó un baremo que, por ser una escala convencional de valores utilizada como base para clasificar y ordenar los elementos de un conjunto, permitió evaluar y explicar los resultados obtenidos. (Ver Tabla 1)

Tabla 1. Baremo de interpretación.

\begin{tabular}{cc}
\hline Rango & Categorías \\
\hline $0-3,99$ & Muy bajas competencias \\
$4-7,99$ & Bajas competencias \\
$8-11,99$ & Medianas competencias \\
$12-15,99$ & Altas competencias \\
$16-20$ & Muy altas competencias \\
\hline
\end{tabular}

Fuente: Elaboración propia (2020).

RESULTADOS

Con relación a los resultados de las sinergias del evento (competencias en ciencias naturales las cuales son: competencias cognitivas, competencias procedimentales y actitudinales. En la Tabla 2 y gráfico 1 se muestra que la sinergia con el menor valor en la mediana fueron las competencias cognitivas, con 9 puntos de un máximo de 20, que, al compararse con el baremo de interpretación, refleja que medianamente los estudiantes tienen conocimientos cognitivos referidos a datos, conceptos y principios científicos, igual situación es la de las competencias procedimentales con 10 puntos.

La competencia que mayor valor obtuvo fue la referida a la competencia actitudinal (Saber ser) con 13,75 puntos, lo cual la ubica en una categoría alta y de un importante valor para esta investigación, porque indica que se puede reforzar. Por otra parte, también se detectó que hay unos pocos casos atípicos en las competencias actitudinales, como son los estudiantes identificados como 55 y 12. 
Tabla 2. Estadísticos de las sinergias del evento competencias científicas.

\begin{tabular}{|c|c|c|c|c|}
\hline & & $\begin{array}{c}\text { Puntaje } \\
\text { transformado } \\
\text { de competencias } \\
\text { cognitivas }\end{array}$ & $\begin{array}{l}\text { Puntaje transformado } \\
\text { de competencias } \\
\text { procedimentales }\end{array}$ & $\begin{array}{l}\text { Puntaje transformado } \\
\text { de competencias } \\
\text { actitudinales }\end{array}$ \\
\hline \multirow[t]{2}{*}{$\mathrm{N}$} & Válidos & 102 & 102 & 102 \\
\hline & Perdidos & 0 & 0 & 0 \\
\hline Mediana & & 9,00 & 10,00 & 13,75 \\
\hline Mínimo & & 1 & 2 & 0 \\
\hline Máximo & & 17 & 18 & 20 \\
\hline \multirow[t]{3}{*}{ Percentiles } & 25 & 7,00 & 6,67 & 10,00 \\
\hline & 50 & 9,00 & 10,00 & 13,75 \\
\hline & 75 & 11,00 & 13,33 & 16,25 \\
\hline
\end{tabular}

Fuente: Elaboración a partir de los datos (2020).

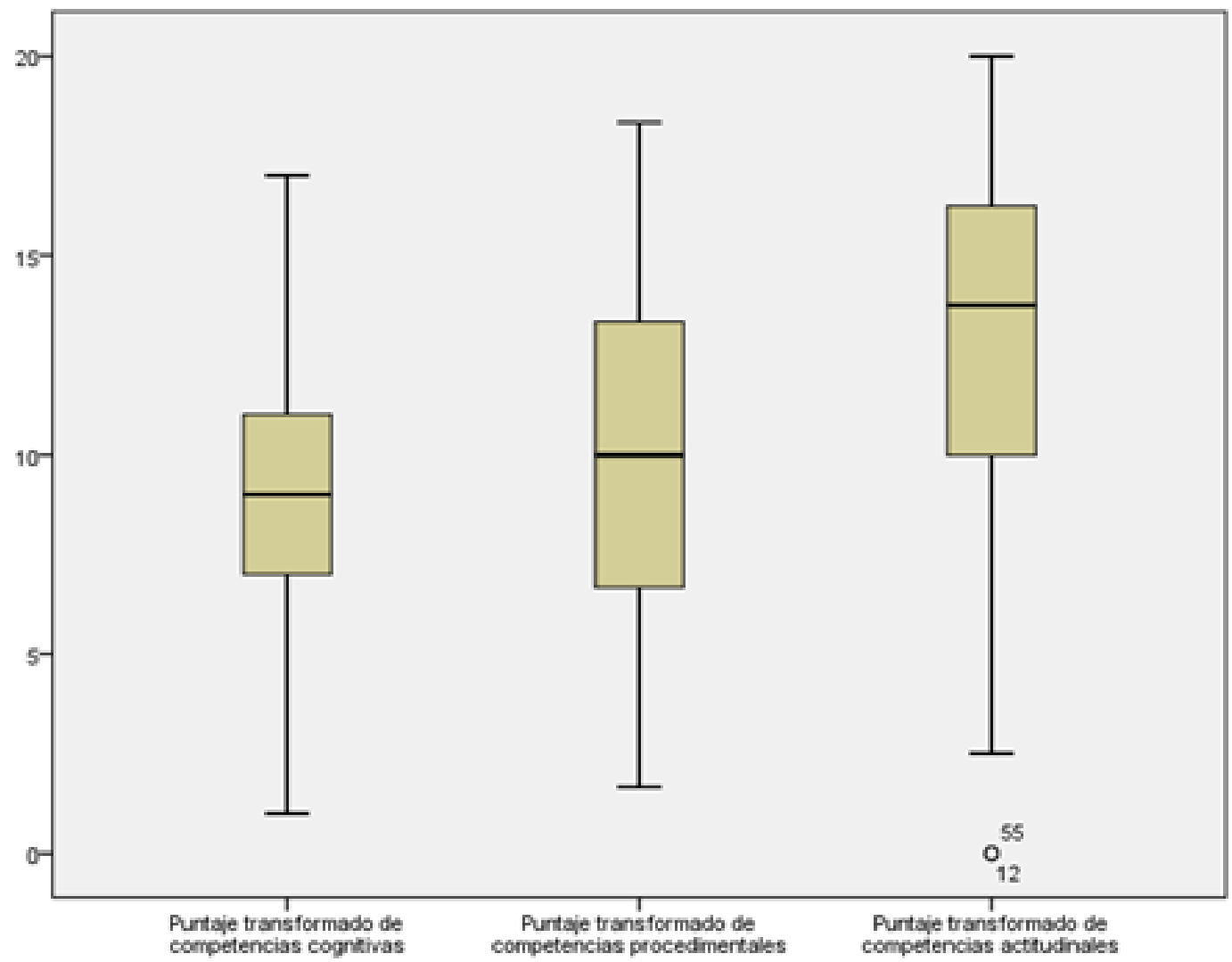

Gráfico 1. Gráfico de caja de bigote de competencias científicas. Fuente: Elaboración a partir de los datos (2010). 
Con respecto a la sinergia de competencias cognitivas, se observa en la gráfica 1 que la distribución es asimétrica. Eso significa que el grupo de estudiantes es heterogéneo; es decir, presenta características dispersas o diferentes. En esa sinergia según indica la Tabla 2, se obtuvo una mediana de 9 puntos, lo cual hace que se ubique en la categoría de medianas competencias. La Tabla 2 de igual manera muestra un puntaje mínimo de 1 punto lo que indica que en el grupo hay estudiantes que obtuvieron solo ese valor, en la prueba de conocimiento aplicada, mientras que quien obtuvo el mayor valor se ubicó en 17 puntos, de un máximo de 20 . El percentil 75 con 11 puntos indica que el $75 \%$ del grupo apenas tiene el 55\% de los conocimientos referidos al nivel cognitivo que deberían tener los estudiantes de los grados 4to y 5 to.

Asimismo, la Tabla 3 presenta cómo se distribuye la población estudiada con relación a las categorías de la sinergia competencias cognitivas, donde se observa que el $45 \%$ se ubica en medianas competencias, $34 \%$ en muy bajas y bajas competencias, $20,6 \%$ en altas y muy altas competencias. Cifras nada favorables para la formación de estudiantes en el área de ciencias.

Tabla 3. Categorías de competencias cognitivas.

\begin{tabular}{|c|c|c|c|c|c|}
\hline & & Frecuencia & Porcentaje & $\begin{array}{l}\text { Porcentaje } \\
\text { válido }\end{array}$ & $\begin{array}{l}\text { Porcentaje } \\
\text { acumulado }\end{array}$ \\
\hline \multirow[t]{6}{*}{ Válidos } & Muy bajas competencias & 3 & 2,9 & 2,9 & 2,9 \\
\hline & Bajas competencias & 32 & 31,4 & 31,4 & 34,3 \\
\hline & Medianas competencias & 46 & 45,1 & 45,1 & 79,4 \\
\hline & Altas competencias & 19 & 18,6 & 18,6 & 98,0 \\
\hline & Muy altas competencias & 2 & 2,0 & 2,0 & 100,0 \\
\hline & Total & 102 & 100,0 & 100,0 & \\
\hline
\end{tabular}

Fuente: Elaboración a partir de los datos (2020). 
Con respecto a la sinergia competencias procedimentales referidas a la forma cómo el estudiante pone en acción sus conocimientos científicos, mediante alguna operación, manipulación o manejo de algún elemento; por ejemplo, leer y comprender textos, decodificar gráficas, organizar información, aplicar algún instrumento, elaborar cuadros o Tablas, así como realizar alguna experimentación, comunicar sus ideas y conocimientos.

En esa sinergia según indica la Tabla 2, se obtuvo una mediana de 10 puntos, que de acuerdo al baremo de interpretación la ubican en la categoría de medianas competencias. El puntaje mínimo de 2 puntos indica que en la prueba algunos estudiantes obtuvieron esa puntuación, y el puntaje máximo fue de 18. La distribución es asimétrica, lo cual indica que el grupo presenta características diferentes en esta sinergia.

Es importante resaltar que esta sinergia se refiere a las competencias del hacer; es decir, requiere una actividad más palpable, visible e inmediata, por lo que es un aspecto que llama la atención a los estudiantes, debido a su edad, ya que les permite involucrarse de forma directa en el quehacer científico, además el dinamismo en el cumplimiento de la tarea, demanda mayor acción del joven.

En la Tabla 4 se presenta cómo se distribuye la población estudiada con relación a las categorías de la sinergia competencias procedimentales, donde se observa que el $44,1 \%$ se ubica en medianas competencias, $28,4 \%$ en muy bajas y bajas competencias, $23,5 \%$ en altas y sólo el 3,9 en muy altas competencias. Estos resultados inducen a afirmar que los estudiantes, a pesar de que se involucran con agrado en actividades procedimentales, presentan dificultades, ya que para ello deben tener competencias cognitivas, en las cuales tienen bajos conocimientos.

Tabla 4. Categorías de competencia procedimentales.

\begin{tabular}{|c|c|c|c|c|c|}
\hline & & Frecuencia & Porcentaje & $\begin{array}{l}\text { Porcentaje } \\
\text { válido }\end{array}$ & $\begin{array}{l}\text { Porcentaje } \\
\text { acumulado }\end{array}$ \\
\hline \multirow[t]{6}{*}{ Válidos } & Muy bajas competencias & 8 & 7,8 & 7,8 & 7,8 \\
\hline & Bajas competencias & 21 & 20,6 & 20,6 & 28,4 \\
\hline & Medianas competencias & 45 & 44,1 & 44,1 & 72,5 \\
\hline & Altas competencias & 24 & 23,5 & 23,5 & 96,1 \\
\hline & Muy altas competencias & 4 & 3,9 & 3,9 & 100,0 \\
\hline & Total & 102 & 100,0 & 100,0 & \\
\hline
\end{tabular}

Fuente: Elaboración a partir de los datos (2020). 
Con respecto a la sinergia competencias actitudinales, se observa en Tabla 2 una mediana de 13,75 puntos, que la resaltan como la mejor competencia que los estudiantes presentan, y hace que se ubique en una categoría de valores altos. Es necesario explicar que esta competencia se refiere a la disposición que los estudiantes encuestados, tienen hacia el aprendizaje de las ciencias y el desarrollo de competencias científicas.

Sin embargo, también se presentan casos atípicos como son el 55 y el 12 con 0 puntos. Evidentemente, estos estudiantes poseen debilidades a nivel general en todas las asignaturas, su desempeño en comparación al resto de sus compañeros es bajo, tienen problemas también en otras áreas como lectura y escritura, lo que les dificulta realizar hasta las actividades más básicas como expresarse y, lógicamente evidencian también desinterés por las ciencias. Esta situación se podría explicar con el poco acompañamiento que reciben en el hogar.

Con relación a la distribución de la población en las categorías de las competencias actitudinales, ésta se muestra en la Tabla 5, donde se observa que el $61,8 \%$ de los estudiantes, se ubicaron en alta y muy alta disposición por aprender los contenidos de ciencia, un 19,6\% en mediana y $18,6 \%$ en baja y muy baja.

Tabla 5. Categorías de competencias actitudinales.

\begin{tabular}{|c|c|c|c|c|c|}
\hline & & Frecuencia & Porcentaje & $\begin{array}{l}\text { Porcentaje } \\
\text { válido }\end{array}$ & $\begin{array}{l}\text { Porcentaje } \\
\text { acumulado }\end{array}$ \\
\hline \multirow[t]{6}{*}{ Válidos } & Muy bajas competencias & 3 & 2,9 & 2,9 & 2,9 \\
\hline & Bajas competencias & 16 & 15,7 & 15,7 & 18,6 \\
\hline & Medianas competencias & 20 & 19,6 & 19,6 & 38,2 \\
\hline & Altas competencias & 33 & 32,4 & 32,4 & 70,6 \\
\hline & Muy altas competencias & 30 & 29,4 & 29,4 & 100,0 \\
\hline & Total & 102 & 100,0 & 100,0 & \\
\hline
\end{tabular}

Fuente: Elaboración a partir de los datos (2020).

\section{DISCUSIÓN}

Después de obtenidos los datos aportados de las respuestas de los estudiantes y analizados los resultados, uno de los primeros elementos que sobresalen y llaman poderosamente la atención para esta investigación, es que de las 40 preguntas formuladas sobre las tres (3) competencias estudiadas, sólo se obtuvo resultados positivos o favorables en cuatro (4) de ellas. Dos correspondientes a lo cognitivo sobre "La acción de la fuerza para desplazar un cuero" y dos (2) referentes a las competencias procedimentales, que tratan sobre "Observar y relacionar patrones de los datos".

Esos resultados alertan sobre muchos elementos a modificar, para generar cambios que mejoren el proceso enseñanza aprendizaje en el área de ciencias naturales. Entre ellos, revisar 
las estrategias aplicadas en el aula, modificar la planificación académica, incluir actividades que estimulen la participación y la motivación de los estudiantes. En síntesis, cambiar la dinámica del hecho educativo.

Del mismo modo, se pudo determinar que los estudiantes que lograron los puntajes más altos, apenas cubren el $75 \%$ de las competencias que deberían tener en estos grados, lo que quiere decir que se está incumpliendo con el perfil definido por el Ministerio de Educación en Ciencias (1998), porque este organismo declara que los estudiantes de los grados cuarto y quinto de primaria se encuentran en un nivel de formación escolar, en donde ya deben ser capaces de construir teorías acerca de los procesos químicos, físicos y biológicos.

Declaración que también se relaciona con lo expuesto en el documento emanado por el MEN (2004, p. 16 y 17), como Estándares Básicos de Competencias. Pautas básicas para esta investigación, porque en ellos literalmente se expone que los estudiantes de los grados cuarto y quinto, al terminar el ciclo de formación, deben saber (Cognitivo) y saber hacer (Procedimental) lo siguiente:

- Identificar estructuras de los seres vivos, que les permitan desarrollarse en un entorno $y$ que pueden utilizar como criterios de clasificación.

- Ubicarse en el universo y en la tierra e identificar características de la materia, fenómenos físicos y manifestaciones de la energía en el entorno.

- Identificar transformaciones en su entorno a partir de la aplicación de algunos principios físicos, químicos y biológicos que permiten el desarrollo de tecnologías.

De igual manera, al contar la población sólo con el $75 \%$ de las competencias definidas para el grado en estudio, es más que determinante para explicar y esperar que los resultados en las pruebas "Saber" sean bajos, tal como se muestra en el informe del Icfes, sobre las evaluaciones presentadas en los años 2014 y 2016, en donde el $67 \%$ y $65 \%$ de los estudiantes de la institución respectivamente al año, se ubican en un nivel mínimo de desempeño en ciencias naturales.

Estos resultados concuerdan con lo planteado por Gallego y otros (2008), quienes afirman que la noción de ciencias que tienen la mayoría de los niños al terminar la primaria es mínima, por no decir nula. Esto también es ratificado por Unesco (2009) cuando afirman que después de cursar los seis años de educación primaria, aproximadamente el $80 \%$ de los estudiantes no logra desarrollar capacidades fundamentales para la comprensión de la ciencia, tales como modelar, inferir, explicar y generar hipótesis.

Por otro lado, es importante tener en cuenta que, en muchas ocasiones, durante las pruebas escritas los estudiantes leen las preguntas sin comprender su contenido, la idea central o el objetivo del texto; por lo que, a pesar de tener el conocimiento no contestan de manera adecuada. Esta situación se explica al revisar las palabras de Campanario y Moya (1999), quienes mencionan que, para el análisis inicial de un problema, el estudiante debe crear un modelo mental relativo a la situación que describe el enunciado.

En otras palabras, no se puede responder una interrogante sin pasar previamente por un proceso capacitante de comprensión e interpretación, para contrastar, analizar, y disponer luego de las herramientas y los conocimientos suficientes para argumentar; así como contar con información suficiente que permita a los estudiantes ubicarse en el contexto y la realidad planteada. Es decir, si no se comprende un postulado en su total complejidad es imposible dar una respuesta razonada.

En cuanto a las competencias cognitivas los resultados muestran que, en esta sinergia, los estudiantes alcanzaron el menor valor de 
la mediana al obtener 9 puntos de 20, lo que demuestra los bajos conocimientos de los jóvenes en ciencias, e indica que los conceptos trabajados en la escuela, en las clases de ciencias naturales no han sido apropiados por los estudiantes debido a que no los relacionan con su entorno.

Asimismo, con respecto a los estándares básicos definidos por el Ministerio de Educación (2004), éstos tampoco se cumplen en lo referente a las competencias cognitivas del área de ciencia, debido a que, según las respuestas obtenidas de los estudiantes en esta investigación, se puede afirmar que tienen poca capacidad para identificar estructuras de los seres vivos, así como las de la materia, los fenómenos naturales y las manifestaciones de la energía.

De igual manera, algunos estudiantes sólo obtuvieron un punto en la prueba, lo que significa que no tienen conocimientos en ciencias y el estudiante que obtuvo el mayor puntaje fue de 17 sobre 20 . El $75 \%$ de los estudiantes sólo obtuvo 11 puntos de 20 en la prueba de competencias cognitivas, lo que quiere decir que sólo tienen el 55\% de los conocimientos científicos que deberían tener, lo cual es ratificado por Pozo y Gómez (2009), al decir que son muchas las investigaciones donde se muestra que los estudiantes no poseen conocimientos conceptuales.

Estos resultados confirman como la educación centrada en la memorización de conceptos, se convierte en un problema para el desarrollo de las competencias cognitivas. Tobón (2013) ratifica ese planteamiento, al expresar que en la actualidad el volumen de la información que se produce segundo a segundo supera las capacidades humanas para almacenarla en la memoria, por lo cual es necesario formar a los estudiantes en habilidades donde puedan aprender sin necesidad de memorizar.

Esa desviación en la práctica educativa muestra como en Colombia aún se mantiene el modelo educativo tradicional, donde se considera al estudiante simple receptor de información, el docente es el que trasmite datos y el aprendizactúa pasivamente. Continuar con ese modelo lleva a que los estudiantes piensen que lo importante es recordar conceptos científicos. Esa posición, según Campanario y Moya (1999), conduce a que los jóvenes consideren equivocadamente, que el conocimiento científico se basa solamente en ecuaciones y definiciones que deben ser memorizadas y posteriormente repetidas.

Es así como, los conceptos que se aprenden sólo de forma memorística, sin comprender, son olvidados fácilmente, según Pozo (1996 c.p. Pozo y Gómez 2009), por lo cual los estudiantes tienen debilidades en las competencias cognitivas. Según el autor antes mencionado, el principal problema de la falta de competencias cognitivas, son las concepciones previas que tienen los estudiantes, las cuales resultan muy difíciles de modificar.

Las ideas previas delos estudiantes provienen de sus vivencias, de su experiencia personal, familiar y educativas; en síntesis, de la forma como conciben el mundo, puesto que como indica Unesco 2009 los estudiantes de la escuela primaria razonan por medio de lo concreto, lo observable, e interpretan los fenómenos desde un punto de vista antropocéntrico.

\section{CONCLUSIONES}

En función de los resultados del estudio se puede concluir que, en general, los estudiantes de los grados cuarto y quinto de la institución Gabriel García Márquez sede B, no poseen las competencias científicas necesarias acordes con el grado en que se encuentran, lo cual afecta su desempeño académico e incide en los resultados insatisfactorios de las pruebas "Saber".

De igual manera, los resultados del estudio permiten concluir que, aunque los alumnos muestran interés y una actitud positiva para realizar actividades acordes a las competencias 
procedimentales y actitudinales, la ejecución de las mismas se ve afectada por deficiencias en la competencia cognitiva.

\section{REFERENCIAS}

Campanario, J. y Moya, A. (1999). ¿Cómo enseñar ciencias? Principales tendencias y propuestas Enseñanza de las ciencias. Madrid

Castillo, S. y Cabrerizo, J. (2010). Evaluación educativa de aprendizajes y competencias Madrid: PEARSON EDUCACIÓN, S.A.https://s3.amazonaws.com/academia. edu.documents/58924038/evaluacic3b3n_ educativa de_aprendizajes $_{-} y_{-}$ competencias20190416-76083-mdcn01. pdf? response-content-

Gallego, A., Castro, J. y Rey, J. (2008). El pensamiento científico en los niños y las niñas: algunas consideraciones $\mathrm{e}$ implicaciones IIEC. Bogotá: http://cmaps. ucr.ac.cr/rid=1RXS8VTTV-1JVFJRL-3L8/ pensamiento\%20cientifico.pdf

Hernández, C. (2005) ¿Qué son las competencias científicas? Foro Educativo Nacional. Bogotá

Luengo, J. (2004). La Educación como objeto de conocimiento. El concepto de Educación Madrid: https://s3.amazonaws.com/ academia.edu.documents/50399621/1 EducacionConcepto.pdf?responsecontent - disposition $=$ inline $\% 3 \mathrm{~B} \% 20$ filename\%3DLA_EDUCACION_COMO_ OBJETO_DE_CONOCIMIENTO. pdf\&X-Amz-Algorithm=AWS4-HMACSHA256\&X-Amz-Credential=AKIAIW OWYYGZ2Y53UL3 A \%2F 20190904\%2 Fus-east-1\%2Fs3\%2Faws4_request $\& \mathrm{X}$ Amz-Date $=20190904 \mathrm{~T} 151431 \mathrm{Z} \& \mathrm{X}$ A m z - Ex pi r e s $=3600 \& X-A m z-$ SignedHeaders $=$ host $\& \mathrm{X}-\mathrm{Amz}-$ Signature $=\mathrm{d} 8$ 99a57b03384f085a21b03ce972534eb29bc7f4 2256444d080adebaff277afd

MEN (2004) Estándares Básicos de Competencias en Lenguaje, Matemáticas, Ciencias y Ciudadanas. Guía sobre lo que los estudiantes deben saber y saber hacer con lo que aprenden. 1ra edición. MEN, Bogotá. Colombia
MEN, (1998). Serie Lineamientos Curriculares. Ciencias Naturales y Educación Ambiental. Ministerio de Educación Nacional. Bogotá: https://www.mineducacion.gov.co/1621/ articles-89869_archivo_pdf5.pdf

Instituto Colombiano para la Evaluación de la Educación (Icfes). (2016). Informe nacional, SABER $3^{\circ}, 5^{\circ}$ y $9^{\circ}$ Resultados nacionales 2009 - 2014 Bogotá: https://www.icfes.gov.co/ documents/20143/1323329/Resultados\%20 nacionales $\% 20$ saber $\% 203 \% 205 \% 209 \% 20$ 2009\%202014.pdf"

Mora, W. y Parga, D. (2005). Evaluación por competencias y estándares de competencia en el campo de la enseñanza de las ciencias y la educación ambiental Enunciación. Bogotá: https://revistas.udistrital.edu.co/index.php/ enunc/article/view/455/694

Parra R., J. (2018). Las competencias de dirección en educación. Una aproximación a su definición estudio e interrelaciones en un contexto moderno globalizado $y$ complejo Opuntia Brava. https://doaj.org/ rticle/19b926b84db94e9284916e685ea8f29b

Pozo, J. y Gómez, M. (2009). Aprender y enseñar ciencia. Del conocimiento cotidiano al conocimiento científico Madrid: Morata S. L.

Tobón, Sergio. (2013). Formación integral y competencias. Pensamiento complejo, currículo, didáctica y evaluación. Bogotá: ECOE

Unesco Office Santiago and Regional Bureau for Education in Latin America and the Caribbean, (2009). Serce. Aportes para la enseñanza de las Ciencia Naturales Pedagogía y Saberes. Chile: Unesdoc. Biblioteca Digital. https://unesdoc.unesco.org/ark:/48223/ pf0000180275

Vallejo, Sonia. (2014). Las competencias científicas en la política educativa colombiana: privilegio de la perspectiva parcial al estudiar su ensamblaje desde los estudios sociales de la ciencia. Bogotá. Colombia 